Zaponherstellung sicher gestellt ist, ist nunmehr seit einiger Zeit ein regelrechter Betrieb im Gange.

An Hand der Abbildungen sei dis jetzige Einrichtung beschrieben. Durch den unten rechts (Abb. 1 u. 2) in der Ecke befindlichen 3pferdigen Motor ${ }^{7}$ ) für 200 Volt Gleichstrom wird ein Luftkompressor, eine Kolbenpumpe (unten links) betrieben, die die Preßluft in den in seinem oberen 'Teil sichtbaren Druckkessel treibt, wo sie unter einem Druck von etwa 4 Atmosphären steht. Von dem Kessel geht ein Rohr ab, das sich unterhalb der Fensterbänke hinzieht, und das an jedem Fenster zwei Entuahmestellen besitzt, so daß erforderlichen Falles im ganzen 12 Personen gleichzeitig arbeiten können. Von der Rohrleitung führen lange bewegliche Schläuche mit doppelter Gewebeein lage zu den Spritzapparaten (Abb. 3), mit denen der Auftrag bewerkstelligt wird. Das Zapon wird in das zylinderförmige, durch abnehmbaren Deckel verschlossene Gefäß gegeben und durch die feine vorn befindliche Düsenöffnung als äußerst feiner Staub herausgetrieben. Für die Bespritzung schmaler, tiefliegender Stellen stehen kleinere Spritzapparate zur Verfügung, damit die bei Benutzung größerer Spritzapparate sich an den hervorragenden "Stellen leicht bildenden körnigen Abscheidungen vermieden werden. Der sich in dem Tränkungsraum schnell verbreitende feine Zaponstaub wird durch eine größere Ventilationsanlage entfernt.

Durch den oben links sichtbaren 5 pferdigen Motor $^{8}$ ) wird ein Ventilator (oben rechts) betrieben, der durch ein nach vorn stufenweise weiter werdendes Rohr unterhalb der Decke, das trompetenähnliche Öffnungen oberhalb jeder Arbeitsstätte besitzt, den Staub ins Freie befördert. Hinter den Arbeitsstellen können noch große halbrunde Eisenblechschirme (s. Abb. 2) aufgestellt werden, um den Zaponstaub für die Absaugeöffnung zusammen zu halten. Für die Behandlung kleinerer Gipsabgüsse ist (auf der Abb. 1 links) noch eine Art Abzugsschrank mit 2 Arbeitsstellen vorhanden, aus dem durch in seinem Boden angebrachte kreisförmige Öffnungen ebenfalls die Luft abgesaugt wird. Um die Gipsabgüsse bequem von allen Seiten mit dem Bestäuber zu behandeln, werden sie auf drehbare Gestelle gestellt, wodurch vor allem der Vorteil erreicht wird, daß die zu bespritzende Fläche stets nach dem Fenster gewandt werden kann.

Der Auftrag selbst geschieht in der Art, daß zuerst mit einem farblosen Zapon gespritzt wird, da sich herausstellte, daB auf diese Weise die später zu erfolgenden deckenden Überzüge dünner sein können, als wenn sogleich die Farbe benutzt wird. Dabei tritt nämlich leicht ein Durchschlagen der schwarzen Schmutzschicht auf. Der farblose Ubberzug muB dann mindestens 2-3 Tage stehen, ehe der erste Farbauftrag gemacht wird, und auch nach diesem wartet man wieder 2-3 Tage, ehe der zweite und meistens letzte Überzug gegeben wird; nur wenn in seltenen Fällen noch die Schmutzschicht durchschimmert, wird nochmals zaponiert. Die Farbe selbst, deren genaue Zusammensetzung mir bekannt ist, besteht aus Zapon, in dem Lithopon verrieben ist. Um einen etwas wärmeren Farbton zu erhalten, wurde etwas fein gemahlener gelber Ocker hinzugegeben.

Der Eindruck, den die so behandelten Gipsabgüsse machen (siehe Abb. 4, Stücke eines Reliefs vor [unten] und nach [oben] der Behandlung), ist ein vorzüglicher; die kalte weiße Farbe frischer Gipse, die um so unangenehmer ist, je größer die Anzahl der aufgestellten Gipsabgüsse, ist verschwunden, und das warme Gelb wirkt besonders bei einem grau getönten Hintergrund angenehm auf das Auge des Beschauers.

Ein weiterer Vorteil ist die geringere Staubempfänglichkeit, da der Staub sich bei den zaponierten Abgüssen viel leichter durch $\mathrm{Ab}$ blasen entfernen läßt, als bei unbehandelten Gipsen, und schließlich gestattet die Zaponierung sogar ein vorsichtiges Abwaschen mit Wasser und selbst mit Seifenwasser, sofern recht weiche Bürsten benutzt werden.

[A. 191.]

\section{Deutschlands Anteil an der italienischen Boraxindustrie.}

\section{Von Hrrmann Schelenz, Cassel.}

Daß ein Vertreter der deutschen Wissenschaft den Italienern eine Industrie vermittelt hat, die ihnen zeitweise eine Art Handelsmonopol gewährte und auch jetzt noch eine ganz erkleckliche Einnahmequelle darstellt, ist auch bei uns fast vergessen. Es verdient eine kurze Er-

7) Bezugsquelle: Wießner \& Baumann, Berlin S. 14, Stallschreiberstr. 58 .

8) Der demnilchst noch durch einen 6 perdigen ersetzt wird. wähnung, auch um zu zeigen, wie deutsche Chemie, damals übrigens nur von Apothekern gepflegt, schon lange ihre Jünger außer Landes schickte, und wie sie ihrem neuen Vaterlande treue Diener waren. Der aus Cöln gebürtige „Direktor der Apotheke" des Großherzogs

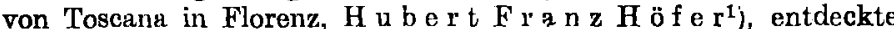
in den sog. Maremmen, Ansammlungen von heißen Flüssigkeiten, wie sie den alten Römern schon als die $\mathrm{A} q$ u a e vo l a te r r a $\mathrm{n}$ a (in der Nähe des heutigen V o l te r ra) aufgefallen waren, und zwar in einer $\mathrm{C} \mathrm{e} \mathrm{r} \mathrm{chi} \mathrm{a} \mathrm{j} \mathrm{o} \mathrm{genannten} \mathrm{Lagone} \mathrm{di} \mathrm{Monte} \mathrm{rotondo} \mathrm{einen}$ Bestandteil, dem sog. Sedativs a I z ähnlich, welches schon im Jahre 1702, wiederum im Grunde ein deutscher Chemiker-Arzt Wil h. Hom berg, aus dem zumeist aus Indien nach Venedig geführten und dort gereinigte $n$, lange bekannten $\mathrm{B}$ or a $x$ abgeschieden hatte.

Es gelang ihm auch, aus ihm durch Zusatz von Natronsalzen B o r a x darzustellen, und damit die Gleichheit beider Stoffe, die später als Bors äu re festgestellt wurden, zu beweisen. Er berichtete im Jahre 1778 in einer, ,Memoria sopra il sa le se dativ o naturale della Toscana" an den Grafen Thun vo $\mathrm{T}$ Wa ls as s in a, die 1781 in einer deutschen Übersetzung herauskam und der gelehrten und der Handelswelt von der neuen Bezugsquelle für den schon damals in der Technik viel benutzten, aber seiner Eigenart nach noch völlig rätselhaften Körper Kunde gab. E r u s t i n $g$ z. B. in seinem „Lexicon Practico-chymicum" von 1770 erklärte noch: „Ich weiß wirklich nicht, was ich von diesem mineralischen festen weißen Crystallinischen und bald wie Allaun anzusehezden Salze sagen soll. Man weiß nicht, ob es ein Compositum oder nur aus einer einfachen Sache gemacht, oder so wie wir es bekommen, vor sich nur ein gereinigtes und crystallsiertes Salz ist. Es ist, nach $N$ o u mann, nicht so leicht zu penetrieren, indem seine Eigenschaften so beschaffen als keines anderen Salzes oder Dinges in der Welt. Es bleibet noch ein Geheimniß, was es wirklich ist. " Daß hundert Jahre früher. J o h a n n S c hröder in seiner "Pharmacopoea medico physica"den B or a x, aus Sal. Ammoniacum, Nitrum, Tartarus calcinatus (Kalium carbonat), G. Arabicum, Sal commune, Mastix, Alumen de roche, in Urin gelöst oder nur aus in Knabenurin gelöstem Sal nitrum durch Auskrystallisieren darstellen läßt, hört sich uns geradezu abentenerlich an.

Nur langsam allerdings traten $H$ ö $f$ e $\mathrm{s}$ Arbeiten aus der Enge seines Laboratoriums in die große Öffentlichkeit und in den Dienst der Technik. Erst im Jahre 1815 wurde eine fabrikartige Darstellung der Borsäure aus den gedachten Dunstquellen begonnen, 1818 wurden nur etwa $25 \mathrm{dz}$ gewonnen. 1828 begann man auf den Vorschlag eines Franzosen Fra n c o is $L_{\text {a }} \mathrm{r}$ d e r e l, zum Dank für die Erhöhung seiner Landeseinkünfte vom Herzoge von Toscana sehr bald zunı Grafen $v$ on $\mathrm{M}$ on te $\mathrm{Cer}$ boli erhoben, die bis $120^{\circ}$ heißen Dämpfe zum Heizen der Abdampfpfannen zu benutzen, und diess die Darstellung unendlich verbilligende Arbeitsart machte die Säure und das daraus dargestellte Boraxsalz erst marktfähig, und dem alten venetianischen, aus indisohem Tinkal u. dgl. dargesteliten erwuchs ein gefährlicher Feind. 1828 sollen $500 \mathrm{dz}$ Borsäure dargestellt worden sein, $18397000,1849100000,1859200000$ usw. 1830 stellte ein Dr. Philip p Strom e yer (vermutlich ein Sohn des Göttinger Professors der Chemie Fri edrio h Strome yer), der vielleicht die Säuredarstellung an Ort und Stelle kennen gelernt hatte (er fand auch bei einem Besuch der Insel V olc a no krystallinische, perlmutterglänzende Schuppen, die der Göttinger, um technische Chemie hochverdiente $S$ trome ye r als Borsëure erkannte $\left.{ }^{2}\right)$ ) in Hamburg, wie Bu chner bezeugt, sehr schönen „raffinierten“ B or a ${ }^{4}$ dar $\left.{ }^{3}\right)$. Inzwischen entdeckte man in allen möglichen anderen Erdteilen, in Californien und sonst noch an der Westküste Amerikas,

1) Er ist fast vergessen. Ich nannte ihn in meiner Geschichte der Pharmazie. Sonst (z. B. in Ernst v. Me yers Geschichte der Chemie) finde ich ihn nicht erwähnt.

2) B u c h n e r, Repertorium 14, 117 [1829]; 35, 429 [1830]. Stromeyor entdeckto auch das Cadmium. Vgl. über ihn $\mathrm{S}$ c h e le nz, Geschichte der Pharmazie.

3) Neben ebenso schönem $S$ a l p et e $r$, übrigens ersteren $z u$ einem Preise von $26 \mathrm{Kr}$. (etwa $80 \mathrm{Pf}$ ) f ür da s Pfund, letzteren zu 26 Fl. den Zentner (ca. $44 \mathrm{M}$ ) ,ohne Berechnung der Füsser". Vielleicht ruht die jetzige blühende Hamburger chemische Industrie auf den Schultern jenes Dr. Ph il i p p und letzten Endes auf den Anregungen des Göttinger Universitätslehrers. Nebenbei möchte ich bemerken, daß um dieselbe Zeit $\mathrm{Natrium} \mathrm{bicarbonat}$ von der Marti u schen Hofapotheke in Erlangen das Pfund zu 2 Fl. $15 \mathrm{Kr}$. (etwa 3,80 M) angeboten wurde. Es war noch ganz ein Erzeagnis der Apothekenlaboratorien. 
in Kleinasien, schließlich im deutschen $S$ t a B f u r t it Borsäure enthaltende Gewässer und Mineralien, die an die Seite derer in Toscana traten und den inzwischen ganz unendlich gesteigerten Bedarf an Borax und reiner Borsäure decken halfen. Immerhin erhielt sich die Fabrikation der beiden Stoffe in Italien nicht nur auf der alten Höhe und brachte dem Lande noch einen reichen Nutzen, sie nahm im Gegenteil weiteren Aufschwung, wiederum durch die Bemühung zweier Deutscher, der Techniker C. M. K u r t"z und $\mathrm{Ph}$. $S \mathrm{chwarzenberg}$, die nach längerem Aufenthalt an den in Betracht kommenden Orten in einer Arbeit: „Die Borsäurefabrikation in Toscana," im Jahre 1874 zu wichtigen Verbesserungen der alten L a r d e r e l schen Heizart Veranlassung gaben. K u r t z war es auch, der zuerst daran dachte, die unter gar nicht unerhebliohen, auf 3-5 Atmosphären geschätzten Druck der Erde entströmenden Dämpfe sofort als Kraft nutzbar zu machen. Erst zwanzig Jahre später wurde die Frage allerdings praktisch gelöst. Einer Dampfmaschine hauchten die Quellen, die das Landvolk, ob ihres noch immer nicht ganz enträtselten Ursprungs jedenfalls sehr lange sçhon mit dem Cerbero, mit dem Wächter der Hölle in Beziehung gebracht hatte, und denen es sich nur unter Abbeten des Rosenkranzes zu nahen wagte, den belebenden Odem ein, der für Italien bei seinem, von den freundschaftlich ihnen verbundenen Engländern vortrefflich ausgenutzten Kohlenmangel zweifellos gerade jetzt aufs angenehmste empfunden wird.

Es würde zu weit führen, hier statistische Zahlen über den Verkehr mit Borax und mehr noch mit Borsäure vorzulegen. Ich möohte nur einige Angaben über die Verwendung der beiden Körper geben. In der metallurgischen Technik scheint Borax lange schon eine Rolle gespielt zu haben, vielleicht waren mit ihm aus seiner fernen östlichen Heimat Nachrichten über seine Verwendbarkeit westwärts gedrungen.

Auf gleiche Quellen kann sebr wohl auch die Verwendung in der Färberei zurückzuführen sein. Der Löwenanteil seines Verbrauchs liegt zweifellos immer noch auf den angeführten gewerblichen $\mathbf{G e}$ bieten. 1821 empfahl weiter G a y - L u s a a c, Holz mit Boraxlösung zu tränken, um es unverbrennlich zu machen. Vielleicht brauchte es das Altertum gelegentlich auch dazu. Der Unterschied zwischen Ala un-Stypteria und sicherlich auch eingeführten natürlichen Borsalzen wird ihm kaum klar gewesen sein. Auch die medizinischen Eigensohaften, die man dem Alaun im Altertum zuschrieb, übrigens auch jetzt noch") beimi (3t, ähneln dem des Borax. Im Jahre 1732 schon entdeckte, wohl bei tappenden Versuchen, ein Ulmer Arzt Le Fève den Boraxweinste in, der sich immer noch bei Blasenleiden eines gewissen Ansehens erfreut. 1830 empfah H ufeland Borax in Lösung als Cosneticum gegen Sommersprossen, Hautröte, in Salbenform gegen Frostbeulen, gegen alle Arten Flechten. 1839 wurde Boraxpulver auch als Mittel gegen die Schwaben oder, wie man sie wegen ihrer Massenüberfälle und ihrer räuberischen Zerstörungswut viel besser nur nennen sollte, die Russen, die B latta o oriental es empfohlen. Seit dem Anfange der 90er Jahre endlich trat B o r s ä u re mit durchschlagendem Erfolg an die Stelle der damals zumeist als Antisepticum benutzten Salicylsäure. Bors ä ureverbands tof $f e, B$ or -

4) Denkt man an die, auch dem in den letzten Jahren wieder warm, von mir übrigens auch als Seifenersatz empfohlenen T o n zukommende Grundlage $A 1 \mathrm{u} \mathrm{m}$ i $\mathrm{n}$ i u $\mathrm{m}$, so darf man sagen, mit Recht. s äu reva selin beherrschen augenblicklich wohl das unsäglioh große Feld der Chirurgie. Daß ihre Leistung eine so überraschend segensvolle ist, ist vermutlich zum guten Tcil dem Stoff zuzusohreiben, dessen genaue Kenntnis und Einführung in den Arzneischatz wiederum, wie aus dem vorstehend Gesagten hervorgeht, des deutschen Barbarenvolks Verdienst ist.

[A. 1.]

\section{Uber die Verwendbarkeit des Shukoffapparates für Molekulargewichtsbestimmungen.}

In dieser Zeitschrift (Angew. Chem. 29, I, 423 [1916]) befindet sich ein Bericht von Georg von Kozicki und $S t$. von Pilat über die Verwendungsmöglichkeit des Shukoffapparates zu Molekulargewichtsbestimmungen. Ich kann die Angaben der Verfasser nur bestätigen, die durch ihre reiches Zahlenmaterial noch besonders wertvoll sind, möchte aber darauf hinweisen, was vielleicht den Verfassern entgangen ist, daß ich schon seit einer längeren Reihe von Jahren den Shukoffapparat für Molekulargewichtsbestimmungen zur Ermittlung der Schmelzpunktdepression benutze. Ich verwendete ihn schon im Jahre 1902 dazu, als ich die vorzügliche Brauchbarkeit des Apparates zur Schmelzpunktbestimmung des Paraffins kennenlernte und beschrieb (Braunkohle 1, 362 [1902]). Ferner benutzte ich ihn zum gleichen Zwecke anläßlich einer Versuchsreihe über die Schmelzpunktserniedrigung von Paraffin (ChemikerZeitung 1904, Nr. 95). An gleicher Stelle wies ich auch darauf hin, da 3 gerade Kohlenwasserstoffe sich nicht einwandfrei als Lösungsmittel bei der Molekulargewichtsbestimmung nach diesem Verfahren eignen, da bekanntlich Lösungsmittel, die nicht nach dem Wassertypus zusammengesetzt sind, bei hydroxylhaltigen Körpern wie Säuren und Alkoholen oft abnorm hohe Molekulargewichte liefern, und aus diesem Grunde brauche ich seitdem als Lösungsmittel die technische Stearinsäure, deren Depressionskonstante durch einen Versuch mit Körpern von bekanntem Molekulargewicht ermittelt wird. Die Stearinsäure hat sich dann für Molekulargewichtsbestimmungen im Shukoffapparat ausgezeichnet bewährt, und unter anderen ist auch das Molekulargewicht einer größeren Reihe von Mineralöldestillaten damit bestimmt worden (vgl. „, Laboratoriumsbuch für die Braunkohlenteerindustrie" Seite 123 und 133; En g le r - H ö f e r, Das Erdöl, Bd. 1, Seite 105/06). Fs sei bemerkt, daß sich der Vakuummantel für den Shukoffapparat nicht als durchaus nötig erwiesen hat. Die Ergebnisse werden dadurch, daß man einen Shukoffapparat mit doppelten Wänden ohne Vakuum verwendet (wodurch der Anschaffungspreis wesentlich erniedrigt wird) kaum beeinträchtigt.

$\mathrm{Da}$ in meinen oben erwähnten Arbeiten die Molekulargewichtsbestimmung mit Hilfe des Shukoffapparates nicht Selbstzweck der Arbeiten war, sondern nur nebenbei als Hilfsmittel bei der Bearbeitung anderer Fragen diente, ist das Verfahren anscheinend der Aufmerksamkeit der beiden Autoren entgangen. Vielleicht dient ihre erneute Anregung dazu, dem Verfahren, das man im wesentlichen als eine Molekulargewichtsbestimmung mit einem vereinfachten $\mathbf{E}$ $\mathbf{k}$ m a n n schen Apparat ansehen kann, weitere Freunde zu erwerben.

Dr. Ed. Graefe, Dresden.

[Zu A. 165.] 